\begin{tabular}{|c|l|}
\hline Title & $\begin{array}{l}\text { Delayed esophageal reconstruction for aortoesophageal fistula caused by an aortic arch aneurysm with microvascular } \\
\text { anastomosis of the left gastric artery and vein. }\end{array}$ \\
\hline Author(s) & Noji, T.; O Kushiba, S.; Kitashiro, S.; Kawarada, Y.; Shichinohe, T.; Kondo, S.; Katoh, H. \\
\hline Citation & $\begin{array}{l}\text { Diseases of the Esophagus, 18(3), 204.206 } \\
\text { https://doi.org/10.1111ग.1442-2050.2005.00481.x }\end{array}$ \\
\hline Issue Date & 2005 \\
\hline Doc URL & http://hdl.handle.net/2115/16959 \\
\hline Rights & The definitive version is available at www.blackwell-synergy.com \\
\hline Type & article (author version) \\
\hline File Information & DE18 3.pdf \\
\hline
\end{tabular}

Instructions for use 
Short code: DES

Title: Diseases of the Esophagus

ISSN: 1120-8694

Created by:

Word version: 7.0a

BEES version: Version 2.42

Email proofs to: kondows@med.hokudai.ac.jp

Copyright: 2005 ISDE

Volume: 18 (Issue: 3)

Cover year: 2005 (Cover month: August)

Article no.: 481

DOI: 10.1111/j.1442-2050.2005.00481.x

Article type: CR (Case Report)

Figures: 2; Tables: 0; Equations: 0; References: 8; Words: 1077; First Page: 000; Last Page: 000

Short title running head: Reconstruction for aortoesophageal fistula

Authors running head: Diseases of the Esophagus

Address correspondence to: Satoshi Kondo, MD, Department of Surgical Oncology, Hokkaido University Graduate School of Medicine, N15W7 Kitaku Sapporo City, Hokkaido, 060-8638, Japan. Email: kondows@med.hokudai.ac.jp

Case report

\section{Delayed esophageal reconstruction for aortoesophageal fistula caused by an aortic arch aneurysm with microvascular anastomosis of the left gastric artery and vein}

T. Noji, S. Okushiba, S. Kitashiro, Y. Kawarada, T. Shichinohe, S. Kondo, H. Katoh

Departments of Surgical Oncology, Hokkaido University Graduate School of Medicine, Sapporo, Japan

SUMMARY

We report a case of aorto esophageal fistula (AEF) with delayed esophageal reconstruction employing microvascular anastomosis. We demonstrate here that our method is useful for delayed esophageal reconstruction following AEF.

KEY WORDS: aortoesophageal fistula, esophageal reconstruction, microvascular anastomosis.

\section{INTRODUCTION}

Aortoesophageal fistula (AEF) is a rare entity that is most often fatal. There is currently no consensus on whether primary repair or esophageal resection is the best therapeutic option for the esophageal defect associated with aortoesophageal fistula.

Recent advances in surgical technique have led to survival in cases of aortoesophageal fistula (AEF), a rare, previously universally fatal condition. The keys to the successful treatment in AEF are control of bleeding, management of the contaminated mediastinum, and delayed esophageal reconstruction. ${ }^{1}$ In this case report, we describe the management of a case of AEF with staged esophageal reconstruction using a gastric tube and microvascular anastomosis.

\section{CASE REPORT}

A 67-year-old woman with a 2-day history of back pain vomited a large amount of blood in October 2001. She was brought to a local hospital in shock. Emergency endoscopy and chest computed tomography (CT) led to a diagnosis of AEF caused by a distalaortic-arch aneurysm (Fig. 1). The patient was transferred to our hospital, and an emergency operation was performed. We performed cardiopulmonary bypass and selective cerebral perfusion, and then replaced the distal aortic arch and the left subclavian artery with a Gelseal one-branch graft. Due to severe inflammation, primary closure of the esophagus was considered unwise. Segmental esophagectomy with cervical esophagostomy and gastrostomy were performed. To prevent graft infection, a free omental flap was raised and inserted into the thoracic cavity through the esophageal hiatus to wrap the graft(Fig. 2A).

コメント [Q1]: Typesetter: insert Figure 1 near here

コメント [Q2]: Typesetter: insert Figure 2 near here 
The esophagus was reconstructed 4 months later. Since the right gastroepiploic artery (RGEA) had been detached in the first operation, the upper stomach was supplied primarily by the left gastric artery (LGA), thus precluding esophageal reconstruction by gastric tube using the greater curvature of the stomach. We decided to mobilize the entire stomach and perform a microvascular anastomosis. After laparotomy, the LGA and left gastric vein (LGV) were transected at their origin and confluence, and a gastric tube was mobilized and placed along a presternal route. To avoid tension, we decided to use the third internal thoracic artery and vein as recipient vessels. These vessels were anastomosed end-to-end to the LGA and LGV by our plastic surgeon colleagues.

After completion of the anastomosis, the color of the gastric tube was good, and esophagogastric continuity was restored (Fig. 2B). The postoperative course was uneventful. The patient was discharged on the 30th postoperative day, approximately 5 months after the first operation. The patient is alive and well at the time of this report.

\section{DISCUSSION}

For distal aortic arch aneurysm, a only few cases using endovascular treatment with stent graft had been reported prior to October 2001. We considered using an endovascular procedure with a stent graft for the present case; because the patient had also a left subclavian artery aneurysm, we opted for graft replacement and segmental esophagectomy.

For an esophageal surgeon, determination of the ideal time for esophageal repair and the method to be used, whether it is primary closure, or resection and reconstruction, are important questions.

To prevent infection of the mediastinum and graft, we raised a free omental flap fed by the RGEA and inserted it into the thoracic cavity. Previous reports have shown that the omentum is very effective in managing an infected surgical field. ${ }^{2}$ In our case, the transfer of a viable omental flap into the potentially infected thoracic cavity and wrapping it around the graft potentially prevented infection of the mediastinum and graft, but it also made delayed reconstruction more difficult.

Some authors have performed esophageal reconstruction in a single stage by placing an omental pedicle graft in the mediastinum and running the reconstructed esophagus through the posterior mediastinum with anastomosis in the neck. ${ }^{3,4}$ This method does not lead to detachment of the RGEA, but possibly increases the risk of anastomotic leakage from the site of anastomosis. Therefore, we performed staged esophageal reconstruction.

In a previous case report of AEF, Tokuda et al. described reported delayed reconstruction using the colon rather than the stomach. This method does not require augmented blood supply from the RGEA, permitting omental transfer. ${ }^{6}$

Using a gastric tube for esophageal reconstruction has significant advantages over other methods, including a lower degree of surgical invasiveness and maybe a lower risk for postoperative complication. Because the upper stomach was supplied only by the LGA, we could not construct a gastric tube using the greater curvature of the stomach. Instead, we mobilized the entire stomach along a presternal route and performed a micro vascular anastomosis. This method worked well and the vascular anastomoses were performed in less than one hour by plastic surgeons.

Microvascular anastomosis for esophageal reconstructions is now considered a routine and safe method, but to our knowledge, there have been no reports of delayed reconstruction for AEF including such a method. ${ }^{7,8}$ We believe delayed esophageal reconstruction with microvascular anastomosis of the left gastric artery and vein for AEF is a viable therapeutic option.

\section{REFERENCES}

1 Snyder D M, Crawford E S. Successful treatment of primary aorto-esophageal fistula resulting from aortic aneurysm. J Thorac Cardiovasc Surg 1983; 85: 457-63.

2 Coselli J J, Crawford E S. Primary aortoesophageal fistula from aortic aneurysm: Successful surgical treatment by use of omental pedicle graft. J Vasc Surg 1990; 12: 269-77.

3 Reardon M J, Brewer R J, LeMaire S A, Baldwin J C, Safi H J. Surgical management of primary aortoesophageal fistula secondary to thoracic aneurysm. Ann Thorac Surg 2000; 69: 967-70.

4 von Oppell U O, de Groot M, Thierfelder C, Zilla P, Odell J A. Successful management of aortoesophageal fistula due to thoracic aortic aneurysm. Ann Throac Surg 1991; 52: 1168-70.

5 Kato M, Kaneko M, Kuratani T, Horiguchi K, Ikushima H, Ohnishi K. New operative method for distal aortic arch aneurysm: combined cervical branch bypass and endovascular stent-graft implantation. J Thorac Cardiovasc Surg 1999 April; 117: 832-4.

6 Tokuda Y, Matsumoto M, Sugita T, Nishizawa J, Matsuyama K, Yoshida K. Successful repair of an aortoesophageal fistula caused by a thoracic aortic aneurysm: report of a case. Surg Today 2004; 34: 357-9.

7 Inoue Y, Tai Y, Fujita H et al. A retrospective study of 66 esophageal reconstruction using microvascular anastomoses: problems and our method for atypical cases. Plast Reconstr Surg 1994; 94: 277-84.

8 Sekido M, Yamamoto Y, Minakawa H, Sasaki S, Furukawa H, Sugihara T. Use of the 'supercharge' technique in esophageal and pharyngeal reconstruction to augment microvascular blood flow. Surgery 2003 September; 134: 420-4.

Fig. 1 Computed tomography on admission reveals that an aneurysm of the thoracic aorta has perforated the esophagus.

Fig. 2 (A) Schema of the first stage (emergency) operation. At first, we replaced the distal aortic arch and the left subclavian artery with a Gelseal onebranch graft. Then segmental esophagectomy with cervical esophagostomy and gastrostomy were performed. To prevent graft infection, a free omental flap was raised and inserted into the thoracic cavity through the esophageal hiatus to wrap the graft. (RGEA: right gastroepiploic artery). (B) Schema of second stage (esophageal reconstruction) operation. We mobilized the entire stomach and perform a microvascular anastomosis. A gastric tube was mobilized and 
placed along the presternal route, and the third internal thoracic artery (ITA) and vein (ITV) were anastomosed end-to-end to the left gastric artery (LGA) and left gastric vein (LGV). 


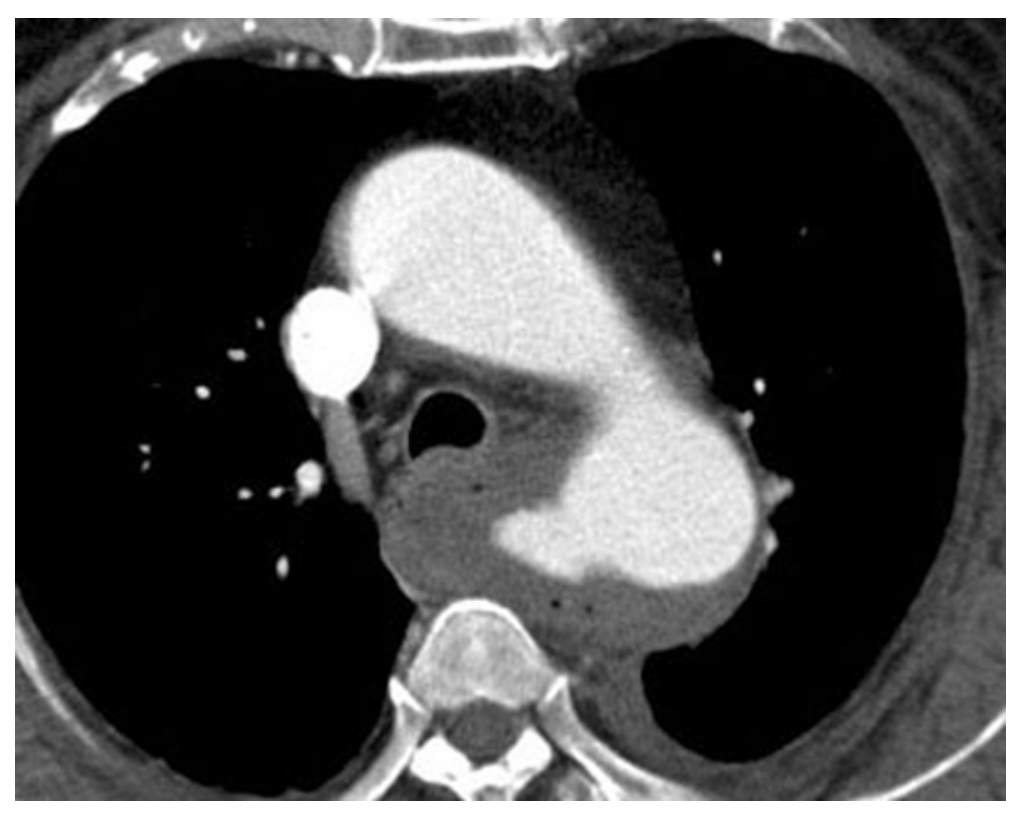




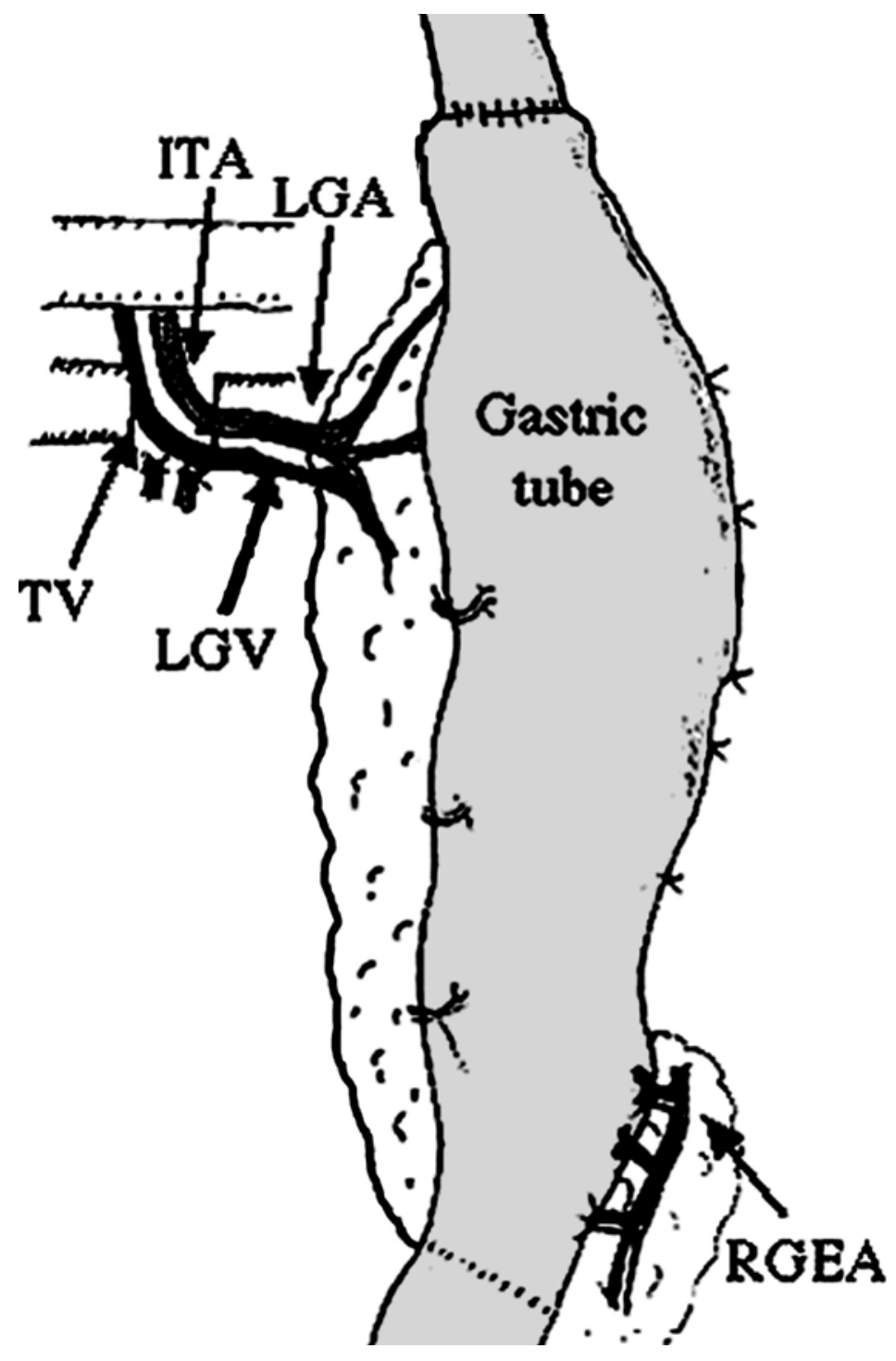

